Historic, Archive Document

Do not assume content reflects current scientific knowledge, policies, or practices. 



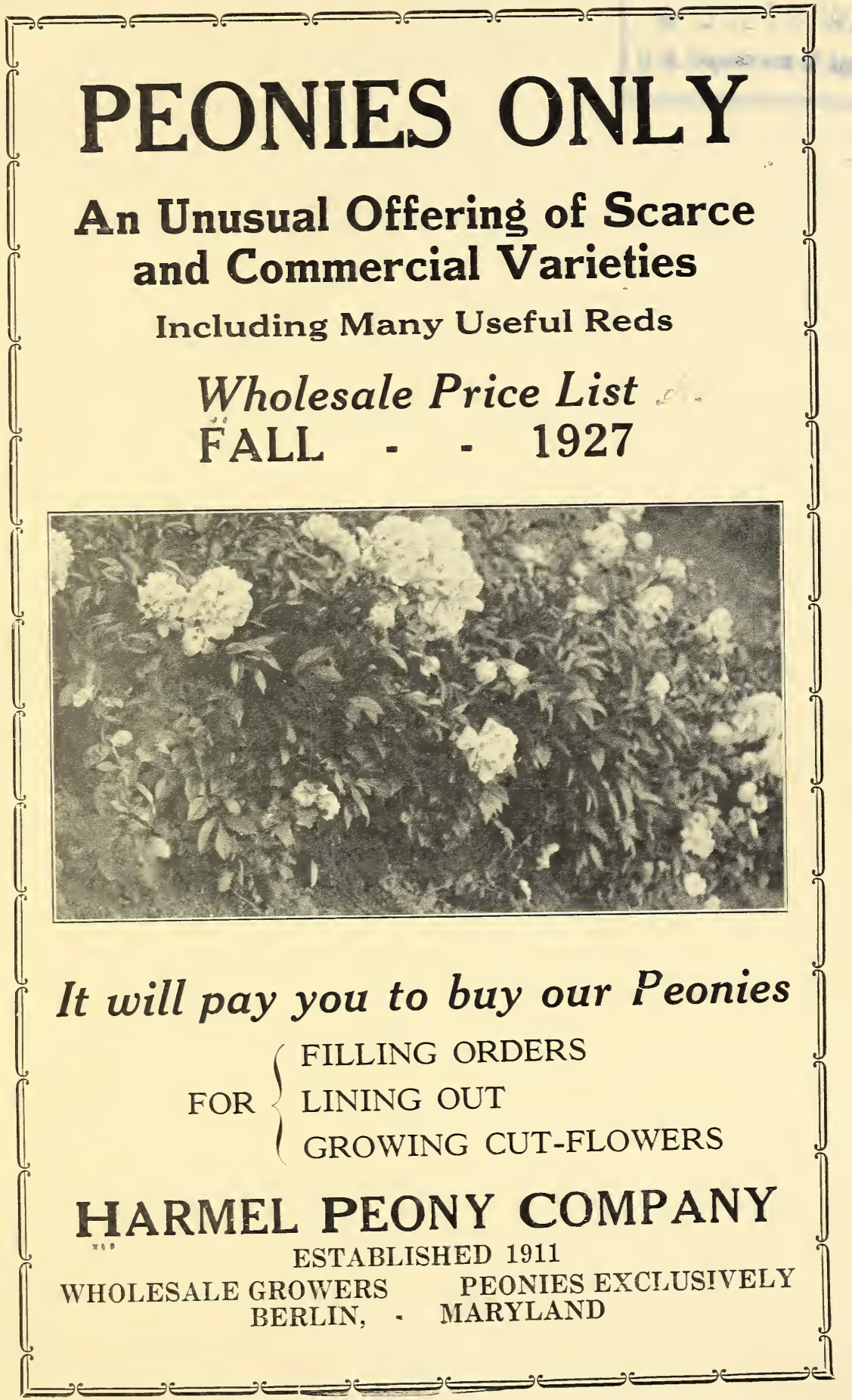




\section{$\underline{\text { Please Read } \text { Before Ordering }}$}

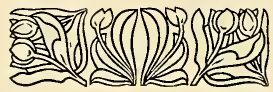

ALL QUOTATIONS subject to stock being unsold upon receipt of order. Prices are fob Berlin, Maryland.

SMALL ORDERS: Our prices are very low and at such prices we cannot fill orders for less than twenty-five of one variety of the lower priced sorts, or five of one variety of the higher priced sorts, except as otherwise quoted.

SIZE OF ROOTS: Our prices are based on liberal, three to five eye divisions, cut from two to three year roots and which should not be confused with divisions cut from old commercial cut-flower clumps that may have lost much of their vitality.

The three to five eye divisions, through years of thorough testing, have proven to be the most satisfactory and economical size to buy and we recommend them to our customers.

Whole clumps quoted on application.

SHIPPING INSTRUCTIONS: When ordering, please give full shipping address when different from postoffice, and state whether you desire shipment via parcels post, express or freight.

PARCELS POST CHARGES: Orders of from one to ten roots, can generally be shipped cheaper by parcels post. Larger orders should go by express or freight. Owing to the multiplicity of Parcels Post rates to the various Parcels Post zones, we request "all cash-in-advance" customers who desire shipment to be made by Parcels Post, to remit fifteen cents extra for the first root and five cents extra for each additional root. With this arrangement, we anticipate that our losses and gains on postage will be about equal.

WHEN WE SHIP: We begin shipping as soon after September first as conditions will permit. Our Peonies are packed in tight, paper-lined boxes with old, damp sawdust and will carry to destination and keep in good condition for an indefinite period when stored in a cold, dry place.

TERMS: Cash with order, less three per cent from individuals or companies not satisfactorily rated. Otherwise, two per cent ten days, net thirty days. Special terms by agreement. All contracts and agreements are made subject to flood, drouth, fire, frost, crop failure or other causes beyond our control.

NON-WARRANTY : We exercise the greatest care to keep our plants true to name and are willing at any time to replace from our own stock, on proper proof, all stock that may prove otherwise, or refund the amount paid, but it is mutually agreed that we shall not at any time be liable for a greater amount than the original cost. We give no warranty expressed or implied, the purchaser assuming all risk and responsibility of transportation, planting, growth, life of stock or any other matter on any stock we sell.

NO COMPLAINTS will be considered that are not made within five days after receipt of stock.

HARMEL PEONY COMPANY. 


\section{The Peony Supreme}

The meteoric rise of the Peony in the esteem of the generai public is without precedent, and it would seem that the time is not far distant when the Peony will displace the rose in its high bid for universal acclaim as our most loved flower.

Aside from the plantings in home gardens and as a part of the perennial border, many hundreds of acres of cut-flower varieties have been planted for the purpose of supplying cut-flowers for home and city trade. Farmers, as well as small truckers, discouraged because of the low prices obtained for ordinary farm crops, are diversifying by planting a part of their acreage to cut-flower varieties of Peonies, and they are finding the venture profitable far beyond their fondest hopes. Florists, because of the insistent demands of customers, are adding Peonies to their assortment of cut-flowers.

\section{"THE LIBERAL SIZE AND FINE QUALITY OF OUR WFIL- BALANCED DIVISIONS, WILL PLEASE YOU."}

TO THE WHOLESALE TRADE, we offer, in quantity, a collection of worth-while varieties of Peonies which are grown under our personal supervivion, on our own eighty acre farm, purchased and maintained solely for the production of fine Peonies on a large scale.

We grow Peonies Only and in our list, we offer "The World's Finest Varieties," including kinds which take the lead among high grade, low priced Peonies in characteristics necessary for producing profitable crops of commercial cut-flowers. We offer to you an opportunity to buy the best at reasonable prices. You will find many real bargains in our list. Other varieties of Peonies are growing in our nurseries and if they are found worthy, they will be offered to our trade as our stock increases.

All varieties are "trued" by experts and our blocks are "rouged" each year in order to locate any possible mixtures in handling.

We sell large orders each year to some of the heaviest buyers of Peonies and we feel that our Peonies will also please you.

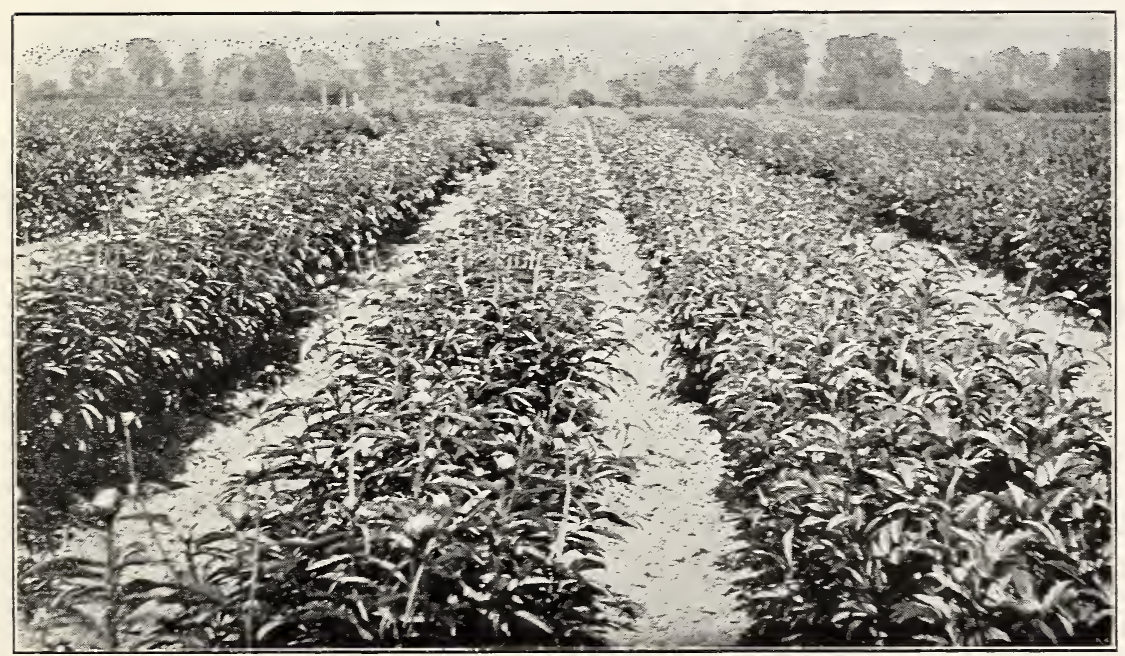




\title{
PEONIES ONLY
}

\section{The World's Finest Varieties}

\section{W \\ HARMEL PEONY COMPANY BERLIN, :-: MARYLAND}

PROFITABLE CUT-FLOWER and other commercial kinds which are especially popular and useful, and which can be offered at a low price, are "underscored" as FESTIVA MAXIMA. While all do not carry the highest ratings, many of them are cut-flower-narket varieties which have withstood the acid test of commercial growing, where none but the most reliable and prolific bloomers are tolerated. Such varieties we endeavor to produce in quantity in order to fill the larger orders of $1,000,5,000$ and 10,000 or more.

\section{General List}

ACHILLE 6.3 (Calot 1855)

Midseason Rose Type Shell Pink

$\left.\begin{array}{ccc}1 & 5 & 25 \\ \text { to } & \text { to } & \text { to } \\ 4 & 24 & 100\end{array}\right\} \begin{array}{r}\text { Divi- } \\ \text { sions } \\ \end{array}$

ADELAIDE E. HOLLIS 7.7 (Hollis 1907) $\$ 2.40 \$ 2.00 \quad$ each

\author{
Midseason Crown type Rose-White
}

AGNES MARY KELWAY 6.0 (Kelway 1898)

Early Midseason Crown Type Deep Violet-Rose

.20 each

ALBA SULFUREA 7.6 (Calot 1860)

Midseason Bomb Type Cream-White

.20 each

ALBATRE 8.7 (Crousse 1885)

Midseason Rose Type Milk White

ALBERT CROUSSE 8.6 (Crousse 1893) .35 each

Late Bomb Type Shell Pink

ALEXANDRE DUMAS 7.1 (Guerin 1862) .18 each

Early Crown Type Light violet-Rose

.25 each

ALFRED de MUSSET 7.9 (Crousse 1885 20 each

Late Rose Type Flesh White

ALICE de JULVECOURT 7.2 (Pele 1857) $\quad .20$ each

Midseason Crown Type Lilac White

$\begin{array}{ccc}\text { ALPHEUS HYATT } 7.4 & \text { (Richardson) } & 2.00 \\ \text { Late } & \text { Rose Type Pink } & \text { each }\end{array}$

PLACE ORDERS EARLY: We do not substitute unless instructed by customer to do so. Money promptly returned for any varieties we are sold out of. 
ALSACE LORRAINE 8.8 (Lemoine 1906)

Late Midseason Semi-Rose Type Cream White

ARCHIE BRAND 7.5 (Brand 1913)

Midseason Bomb Type

Shell Pink

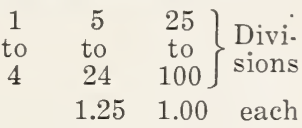

ARMANDINE MECHIN 7.2 (Mechin 1880)

Midseason Rose Type Violet Crimson

ARTEMISE 5.6 (Calot 1861)

Midseason Crown Type Salmon Pink

ASA GRAY 8.1 (Crousse 1886)

Midseason Semi-Rose Type Lilac Pink

$.40 \quad$ each

AUGUSTE LEMONIER 6.3 (Calot 1865)

Midseason Anemone Type Violet Red

AUGUSTE VILLALME 7.3 (Crousse 1895)

Late Rose Type Violet Rose

.30 each

.20 each

.40 each

.20 each

.20 each

AURORE 8.0 (Dessert 1904)

Late Semi-Rose Type Lilac White

AVALANCHE 8.7 (Crousse 1886)

Late Hidseason Crown Type ivory White

AVIATEUR REYMOND 8.0 (Dessert 1915)

Midseason Semi-double Type Cherry Red

1.25 each

BARONESS SCHROEDER 9.0 (Kelway 1889)

Late Rose Type Flesh White

BAYADERE 8.5 (Lemoine 1910)

Midseason Rose Type

Cream White

$3.20 \quad 2.75$

each

BEATRICE KELWAY 7.0 (Kelway)

Rose Pink

.20 each

BEAUTE de VILLECANTE 7.2 (Gombault 1856) Late Midseason Crown Type Violet Rose

BEAUTY'S MASK 7.9 (Hollis 1904)
Late Crown Type

BELLE HOUGH 7.6 (Terry) Late Rose Type Silvery Rose

BERLIOZ 7.0 (Crousse 1886)

Late Midseason Rose Type Currant Red

.30 each

.25 each

.40 each

(2)

.20 each

$.50 \quad$ each

$.20 \quad$ each

.20 each

.20 each

BERNARD PALISSY 6.7 (Crousse 1879)
Late
Rose Type

BOULE DE NEIGE 8.0 (Calot 1867)

Early Midseason Semi-rose Type White,

25 each

Sulphur Center

BUNKER HILL 7.3 (Hollis 1906)

Midseason Rose Type

Tyrian Rose

$1.50 \quad$ each

LARGE ORDERS: Please submit your "want list." On certain items, in quantity, it is often possible for us to quote lower than printed prices. 
CANARI 7.0 (Guerin 1861) Midseason Bomb Type

Flesh, Yellow Center

CAPTAIN CHEAPE (Kelway)

Pink and Cream

$\left.\begin{array}{ccc}1 & 5 & 25 \\ \text { to } & \text { to } & \text { to } \\ 4 & 24 & 100\end{array}\right\}$ ivivi-

$424 \quad 100\}$ sions

$2.50 \quad 2.00$

each

CARMEN 7.2 (Lemoine 1898)

Midseason Semi-rose Type Hydrangea Pink

CARNEA ELEGANS 7.8 (Calot 1860)

Midseason

Crown Type

Flesh Pink

CARNEA ELEGANS 7.1 (Guerin 1850)

Midseason Rose Type Hydrangea Pink

CARNEA TRIUMPHANS 6.3 (Guerin 1852)

Midseason Crown Type Pale Pink

CAVALLERIA RUSTICANA 7.3 (Kelway)

Midseason Semi-rose Type Violet Crimson

CHARLEMAGNE 5.3 (Crousse 1880)

Late Rose Type Lilac White

CHARLES VERDIER (Unknown)

Late Rose Type Purplish Light Carmine

CHARLOTTE CUSHMAN 7.6 (Hollis 1904)

Late Rose Type Solferino Red

$1.00 \quad$ each

CHESTINE GOWDY 8.4 (Brand 1913) 1.00 each

Late Midseason Crown Type Shell Pink

CHRISTINE SHAND (Kelway)

Delicate Flesh

CLAIRE DuBOIS 8.7 (Crousse 1886)

Late Midseason Rose Type Satiny Pink

CLEMENTINE GILLOT 6.9 (Crousse 1885)

Late Crown Type Tyrian Rose

.25 each

20 each

.20 each

.20 each

.20 each

.20 each

.25 each

CONSTANT DEVRED 7.5 (Calot 1868)

Very Late Rose Type Dark Carmine

COQUELIN 7.2 (Dessert 1905)

Early Semi-double Type Tyrian Rose

$1.50 \quad 1.00$ each

.40 each

.20 each

$.60 \quad .50$ each

.25 each

.20 each

$\begin{array}{cc}\text { COURONNE d'OR } & 8.1 \text { (Calot 1873) } \\ \text { Late Midseason } & \text { Semi-rose Type Snow White }\end{array}$

DAYBREAK 6.8 (Hollis 1910) 1.00 each Early Semi-double Type

DeCANDOLLE 7.2 (Crousse 1880)

Late Midseason Rose Type Currant Red

.20 each

DELACHEI 7.1 (Delache 1856)

Late Midseason Rose Type Violet Crimson

.25 each

PLACE ORDERS EARLY: We do not substitute unless instructed by customer to do so. Money promptly returned for any varieties we are sold out of. 


\begin{tabular}{|c|c|c|c|c|}
\hline & $\begin{array}{l}1 \\
\text { to } \\
4\end{array}$ & $\begin{array}{l}5 \\
\text { to } \\
24\end{array}$ & $\left.\begin{array}{c}25 \\
\text { to } \\
100\end{array}\right\}$ & $\begin{array}{l}\text { Divi- } \\
\text { sions }\end{array}$ \\
\hline $\begin{array}{ccc}\text { DELECOURT VERHILLE } & 6.0 \text { Delecourt } & \text { Verhille 1860) } \\
\text { Midseason } & \text { Bomb Type } & \text { Lilac White }\end{array}$ & & & .20 & each \\
\hline$\frac{\text { DELICATISSIMA }}{\text { Midseason }}^{7.6}$ Rose Type $\quad$ Lilac Rose & & & .20 & each \\
\hline $\begin{array}{ll}\text { DENIS HELYE } & 6.3 \text { (Verdier 1860) } \\
\text { Midseason } & \text { Semi-rose Type }\end{array}$ & & & .20 & each \\
\hline $\begin{array}{l}\text { DIRECTEUR AUBRY } 6.1 \text { (Crousse } \begin{array}{l}\text { 1897) } \\
\text { Late }\end{array} \\
\text { Deep Amaranth }\end{array}$ & .25 & & & each \\
\hline $\begin{array}{l}\text { DOCTEUR BOISDUVAL } 7.6 \text { (Guerin } 1850 \text { ) } \\
\text { Late Midseason Rose Type Violet Crimson }\end{array}$ & .25 & & & each \\
\hline $\begin{array}{lll}\text { DR. BRETONNEAU } & 6.9 \text { (Guerin } & \text { 1850) } \\
\text { Midseason } & \text { Rose Type } & \text { Lilac White }\end{array}$ & & & .18 & each \\
\hline $\begin{array}{l}\text { DR. BRETTONEAU } 6.4 \underset{\text { Berdier 1854) }}{\text { (Varly Midseason }} \quad \text { Bomb Type } \quad \text { Lilac Rose }\end{array}$ & & & .18 & each \\
\hline $\begin{array}{cc}\text { DR. CAILLOT } & 7.2 \begin{array}{l}\text { (Verdier 1856) } \\
\text { Midseason }\end{array} \\
\text { Semi-rose Type }\end{array}$ & & & .20 & each \\
\hline $\begin{array}{ccc}\text { DORCHESTER } & 7.7 \text { (Richardson } & 1870 \text { ) } \\
\text { Very Late } & \text { Rose Type } & \text { Hydrangea Pink }\end{array}$ & & & .20 & each \\
\hline$\frac{\text { DUC de WELLINGTON }}{\text { Late Midseason Bomb Type White, Sulphur Center }}$ & & & .20 & each \\
\hline $\begin{array}{c}\text { DUCHESSE de NEMOURS } 8.1 \text { (Calot 1856) } \\
\text { Early Midseason, Crown Type White, Sulphur } \\
\text { Center }\end{array}$ & & & .18 & each \\
\hline DUCHESS OF ALBANY (Kelway 1915) Deep Pink & & 1.00 & .75 & each \\
\hline DUCHESS OF BEDFORD (Kelway) & & 1.00 & & each \\
\hline $\begin{array}{ccc}\text { DUKE OF DEVONSFIRE } 7.3 & \text { (Kelway 1895) } \\
\text { Late } & \text { Rose Type } & \text { Deep Rose }\end{array}$ & & & .20 & each \\
\hline $\begin{array}{l}\text { EDOUARD ANDRE } 7.1 \text { (Mechin } 1874 \text { ) } \\
\text { Midseason Semi-double Type Velvety Crimson }\end{array}$ & & & .20 & each \\
\hline $\begin{array}{l}\text { EDULIS SUPERBA } \\
\text { Very Early Crown Type }\end{array}$ & & & .18 & each \\
\hline $\begin{array}{l}\text { E. G. HILL } 7.8 \text { (Lemoine 1906) } \\
\text { Early Midseason Semi-rose Type Rose Pink }\end{array}$ & & 1.00 & .75 & each \\
\hline $\begin{array}{l}\text { ELIZABETH BARRETT BROWNING } 9.2 \text { (Brand 1907) } \\
\text { Very Late } \\
\text { Rose Type }\end{array}$ & ) 4.00 & & & each \\
\hline $\begin{array}{l}\text { ELLA CHRISTINE KELWAY } 7.3 \text { (Kelway 1899) } \\
\text { Midseason } \\
\text { Rose Type }\end{array}$ & .25 & & & each \\
\hline $\begin{array}{ll}\text { ELWOOD PLEAS } & 8.7 \text { (Pleas 1900) } \\
\text { Late Midseason } & \text { Rose Type }\end{array}$ & 2.40 & 2.00 & 1.75 & each \\
\hline
\end{tabular}

LARGE ORDERS: Please submit your "want list." On certain items, in quantity, it is often possible for us to quote lower than printed prices. 
EMPRESS EUGENIE (Kelway)

ENCHANTERESSE 8.9 (Lemoine 1903)

$$
\text { Very Late }
$$

Rose Type

ETTA 8.0 (Terry 1905)

Late Rose Type

EUGENE BIGOT 8.3 (Dessert 1894)

Late Midseason Semi-rose Type Brilliant Crimson

EUGENE REIGNOUX 7.3 (Dessert 1905)

Early Midseason Semi-double Type Carmine Pink

EUGENE VERDIER 8.3 (Calot 1864)

Late Rose Type

Flesh Pink

EUGENIE VERDIER 8.6 (Calot 1864) Early

Semi-rose Type

EVANGELINE 8.2 (Lemoine 1910) Midseason Rose Type

EXQUISITE 8.5 (Kelway 1912) Midseason

Semi-double Type

FARIBAULT 7.8 (Brand 1918) Late Rose Type

Cream White

Shell Pink

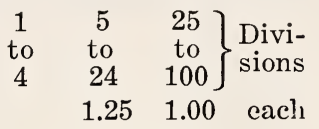

$4.00 \quad 3.50 \quad 3.00 \quad$ each

$.90 \quad .75$ each

$.75 \quad .50 \quad$ each

$1.00 \quad .75$ each

$.75 \quad .50 \quad$ each

.40 each

Shell Pink

Lilac Pink

$2.80 \quad 2.25 \quad 2.00 \quad$ each

$2.40 \quad 2.25 \quad$ each

Cherry Rose

Deep Rose

FELIX CROUSSE 8.4 (Crousse 1881)

\footnotetext{
Midseason Rose Type Ruby Red Most popular of all red Peonies for cut-flower purposes. This variety sold only in assortment with other varieties.
}

FESTIVA 8.0 (Donkelaer 1838)

Midseason Rose Type White

FESTIVA MAXIMA 9.3 (Miellez 1851)

Early Rose Type White

FLAMBEAU 7.1 (Crousse 1897)

Midseason Rose Type Purple Red

FLORAL TREASURE 7.5 (Rosefield 1900)

Early Midseason Rose Type Salmon Pink

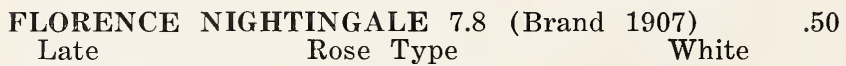

$1.00 \quad .90$ each

.35 each

.20 each

.18 each

.20 each

.20 each

FRAICHEAUR 8.2 (Lemoine 1914)
Rose Type

$\begin{array}{lll}4.00 & 3.50 \quad \text { each }\end{array}$

FRANCES SHAYLOR 8.6 (Shaylor 1915)

Midseason Rose Type White and Yellow

FRANCES WILLARD 9.1 (Brand 1907) Late Midseason Rose Type Blush White each

$2.40 \quad 2.00 \quad 1.50 \quad$ each

PLACE ORDERS EARLY: We do not substitute unless instructed by customer to do so. Money promptly returned for any varieties we are sold out of. 
FRANCOIS ORTEGAT 6.7 (Parmentier 1850) Midseason Semi-rose Type Deep Crimson GALTEE MORE (Kelway)

GEN. de BOISDEFFRE 6.7 (Crousse 1896) Late Bonb Type

Dark Crimson

Lilac Rose

$\left.\begin{array}{ccc}1 & 5 & 25 \\ \text { to } & \text { to } & \text { to } \\ 4 & 24 & 100\end{array}\right\} \begin{gathered}\text { Divi- } \\ \text { sions } \\ \end{gathered}$

$1.00 \quad .90$ each

.20 each

.20 each

.25

each

Rose

GENERAL DODDS 7.2 (Crousse 1893)

Late Semi-rose Type Tyrian Rose

GENERAL JOFFRE (Kelway)

GEORGE HOLLIS 7.7 (Hollis 1907) Late Rose Type

each

GEORGE WASHINGTON 7.2 (Hollis 1904) Midseason Semi-rose Type Dark Crimson

$\begin{array}{llll}\text { GEORGIANA SHAYLOR } 8.9 \text { (Shaylor } & 1908 \text { ) } \\ \text { Midseason } & \text { Rose Type } & \text { Flesh Pink }\end{array}$

GERMAINE BIGOT 8.5 (Dessert 1902)

Midseason Crown Type Lilac Rose

GINETTE 8.6 (Dessert 1915)

Late Midseason

Salmon Flesh

$2.40 \quad 2.25$

.20 each

$\begin{array}{llll}4.00 & 3.25 & 3.00 & \text { each }\end{array}$

.25 each

GISMONDA 8.2 (Crousse 1895) Very Late Rose Type

Flesh Pink

GLOIRE de CHARLES GOMBAULT 7.9 (Gom.- Des.) Midseason Crown Type Rose and Cream

GLOIRE de TOURAINE 7.6 (Dessert 1908) Very Late Rose Type Velvety Crimson

GLORY OF SOMERSET 7.9 (Kelway 1887) Midseason Bomb Type Soft Pink

GOLDEN HARVEST 7.3 (Rosefield 1900)

Midseason Bomb Type Pink and Cream

GOLIATH 7.0 (Hollis 1904)

Midseason Rose Type Tyrian Rose GRACE LOOMIS 9.2 (Saunders 1920)
Rate
Rose Type

Rose Type

White

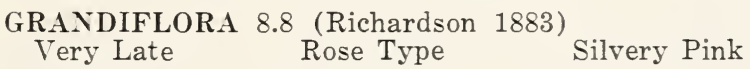

$.60 \quad .50$ each

GRAZIELLA 7.4 (Unknown)

Midseason Rose Type Pink and Salmon

.40 each

.25 each

.20 each

.25 each

.20 each

.20 each

GROVER CLEVELAND 8.2 (Terry 1904)

Late Rose Type Dark Crimson 
HELENA LESLIE (Barr)

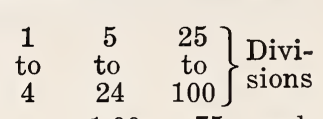

$1.00 \quad .75$ each

White and Sulphur

HELEN WOLAVER 7.9 (Brand 1918)

Very Late Rose Type

Rose Pink

HENRI DEMAY 7.0 (Calot 1866) Midseason Bomb Type

Aniline Red

HUMEI 5.7 (Anderson 1810) Very Late Rose Type

Cherry Pink

$.75 \quad .60$ each

.20 each

.20 each

$\begin{array}{lll}4.00 & 3.00 \quad \text { each }\end{array}$

$8.00 \quad$ each

JAMES BOYD 8.9 (Thurlow 1919)

Late

Rose Type

Flesh Pink

JAMES R. MANN 8.7 (Thurlow 1920) Midseason

Rosy Pink

$12.00 \quad$ each

.20 each

JEANNE d'ARC 7.9 (Calot 1858)

Midseason Crown Type Pink and Cream

JEANNE GAUDICHAU 8.3 (Millet 1902)

Late Rose Type Blush White

JEANNOT 9.2 (Dessert 1918) $\quad 9.60 \quad 8.00 \quad$ each Midseason Rose Type

Lavender Pink

JESSIE SHAYLOR 8.6 (Shaylor 1916)

Midseason

Rose Type

Deep Cream

$6.40 \quad$ each

JOHN HANCOCK 7.6 (Hollis 1907)

Brilliant Crimson

.25

each

.20 each

JULIETTE DESSERT 7.5 (Dessert 1888)

Midseason Rose Type Silvery Crimson

KELWAY'S GLORIOUS 9.8 (Kelway 1909-10) $14.40 \quad 12.00 \quad$ each Late Midseason Rose Type Cream White

KELWAY'S QUEEN 8.8 (Kelway 1909) $\quad 12.00 \quad 10.00 \quad$ each Late Rose Type Flesh Pink

LA COQUETTE 6.8 (Guerin 1861) Midseason Crown Type Creamy Pink

.20 each

LADY ALEXANDRA DUFF 9.1 (Kelway 1902) $3.20 \quad 2.25 \quad$ each Midseason Semi-double Type Delicate Pink

.20 each

$\begin{array}{ll}\text { LADY CURZON } & 7.6 \text { (Kelway 1901) } \\ \text { Early } & \text { Crown Type }\end{array}$

LADY DERBY (Kelway)

LADY STRADBROKE (Kelway 1915)

Rose White

$1.00 \quad .75$ each

Rose Pink $2.50 \quad 2.00 \quad$ each

PLACE ORDERS EARLY: We do not substitute unless instructed by customer to do so. Money promptly returned for any varieties we are sold out of. 
LA FAYETTE 7.8 (Dessert 1904) Midseason Rose Type

LA FIANCEE 7.5 (Lemoine 1898) Midseason Crown Type

LA FONTAINE 8.4 (Lemoine 1904) Late Midseason Bomb Type

LA FRANCE 9.0 (Lemoine 1901) Late Midseason Rose Type Apple Blossom Pink

LA LORRAINE 8.6 (Lemoine 1901) Midseason Rose Type

LAMARTINE 8.4 (Lemoine 1908) Midseason Rose Type

LANGPORT QUEEN (Kelway)

LA PERLE 8.5 (Crousse 1885) Midseason Rose Type

LA ROSIERE 8.3 (Crousse 1888) Midseason Semi-double Type

LA TENDRESSE 8.1 (Crousse 1896) Early Rose Type

Salmon Pink

\begin{tabular}{|c|c|c|}
\hline $\begin{array}{c}1 \\
\text { to } \\
4\end{array}$ & $\begin{array}{c}5 \\
\text { to } \\
24\end{array}$ & $\left.\begin{array}{c}25 \\
\text { to } \\
100\end{array}\right]$ \\
\hline & 1.00 & .75 \\
\hline
\end{tabular}

White

Violet Rose

$\begin{array}{lll}4.80 & 3.50 \quad \text { each }\end{array}$

$6.40 \quad 3.50 \quad$ each

Cream White

$4.80 \quad 4.00 \quad$ each

Rose Carmine

$2.50 \quad 2.00$

each

Rosy Blush

Soft Pink

.40 each

.40 each

Cream White

.30 each

Cream White

.20 each

LA TULIPE 7.5 (Calot 1872)
Late Midseason Semi-rose Type Delicate Pink

L'ECLATANTE 7.7 (Calot 1860)

Midseason Bomb Type Velvety Crimson

LE CYGNE 9.9 (Lemoine 1907) Midseason Rose Type

Milk White

$8.00 \quad 6.00 \quad$ each

.20 each

$\begin{array}{cc}\text { L'INDISPENSABLE } & 7.3 \text { (Unknown) } \\ \text { Late Midseason } & \text { Rose Type }\end{array}$

LIVINGSTONE 8.1 (Crousse 1879)
Late
Rose Type
Rose Pink

LORD ROSEBERY 7.8 (Kelway 1895-6) Midseason Semi-double Type Light Crimson

LOUISA BRAND 7.8 (Brand 1913)

Late Midseason Semi-rose Type Blush White

LOUIS van HOUTTE 6.9 (Calot 1867)

Late Midseason Semi-rose Type Dark Crimson

LOVELINESS 8.8 (Hollis 1907)

Late Rose Type Hydrangea Pink

$\begin{array}{ll}\text { LUCY E. HOLLIS } & 7.8 \text { (Hollis 1907) } \\ \text { Very Late } & \text { Rose Type }\end{array}$

MME. AUGUSTE DESSERT 8.6 (Dessert 1899)

.25 each

.40 each

$.75 \quad .50 \quad$ each

$\begin{array}{lll}4.00 & 3.00 \quad \text { each }\end{array}$

.25 each

$2.80 \quad 2.00 \quad 1.75 \quad$ each

1.25 each

.50 each

LARGE ORDERS: Please submit your "want list." On certain items, in quantity, it is often possible for us to quote lower than printed prices. 


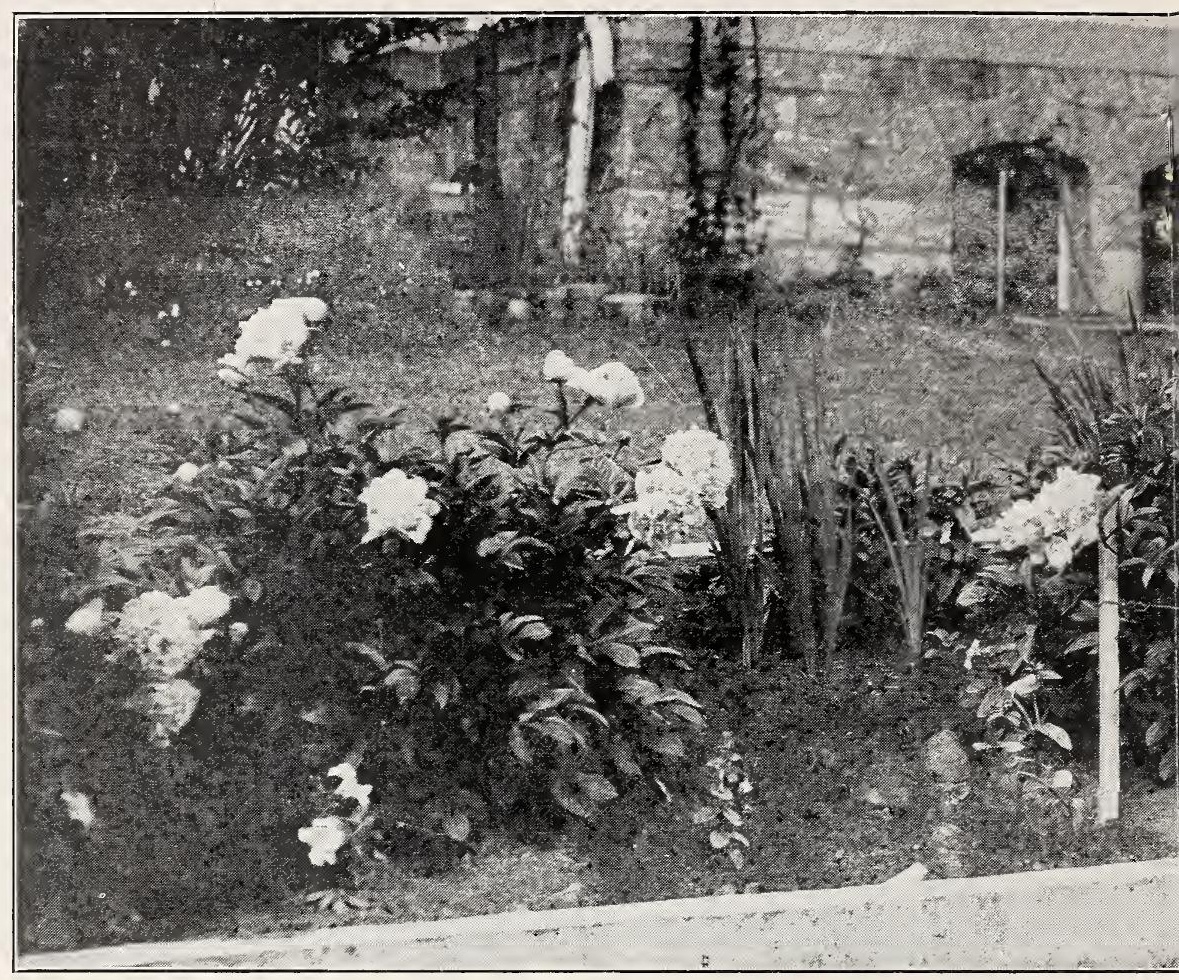

MME. BARILLET DESCHAMPS 7.8 (Calot 1868) Early Midseason Semi-rose Type Violet Rose

MME. BOULANGER 7.8 (Crousse 1886) Late Midseason Rose Type

Light Pink

MME. BREON 7.4 (Guerin 1850) Early Bomb Type

MME. BUCQUET 7.3 (Dessert 1888)

Midseason Semi-rose Type

Dark Crimson

Flesh White

$\left.\begin{array}{ccc}1 & 5 & 25 \\ \text { to } & \text { to } & \text { to } \\ 4 & 24 & 100\end{array}\right\} \begin{array}{r}\text { Divi- } \\ \text { sions } \\ \text { each }\end{array}$

$.75 \quad .50 \quad$ each

.20 each

.30 each

.30 each

20 each

Mauve Pink

Pure White

.20 each

.20 each

Flesh Pink

Lilac White
.20 each
Late Rose Type
MME. de VATRY 7.7 (Guerin 1863)

Midseason

Crown Type
9 (Calot 1866)

Crown Type 


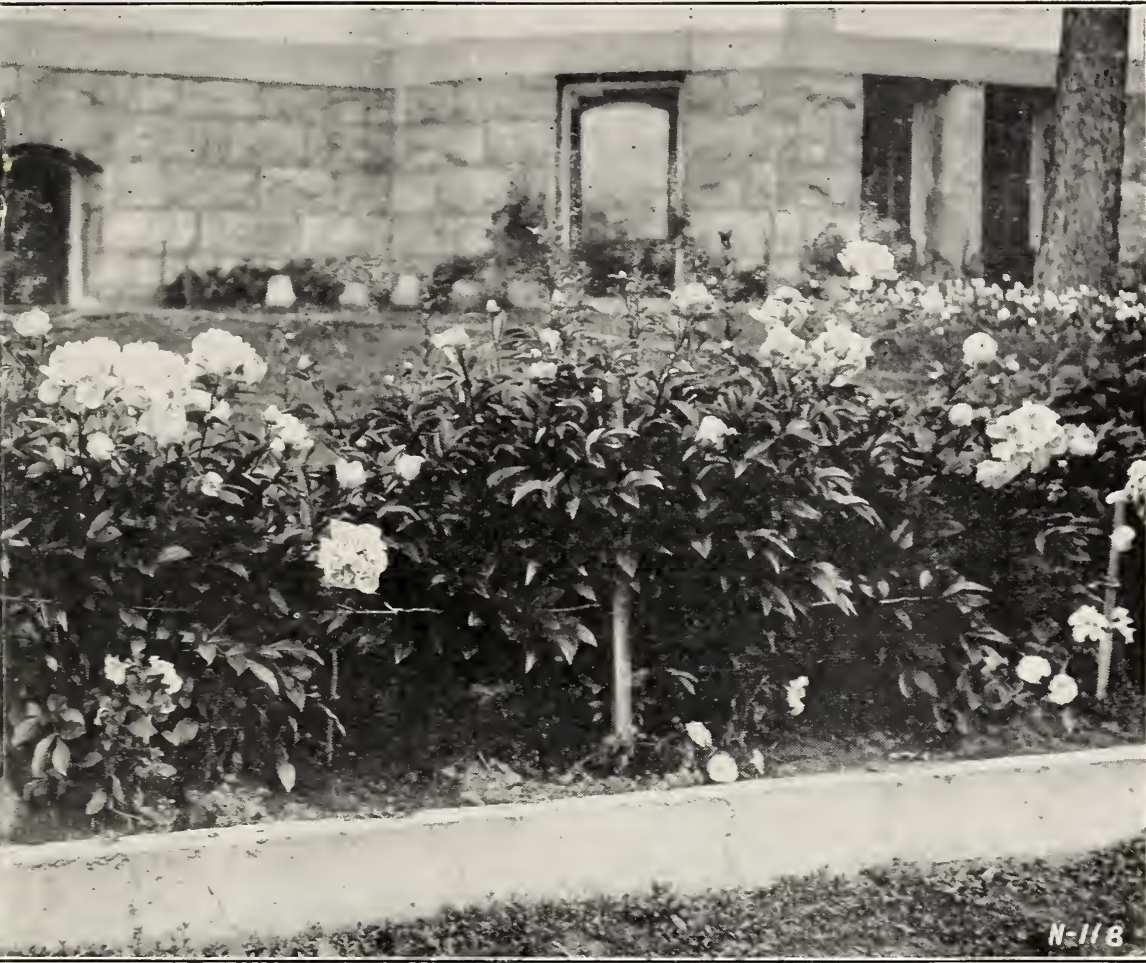

MME. de VERNEVILLE 7.9 (Crousse 1885)

\begin{tabular}{lcr}
\hline Early & Bomb Type & Milk White \\
$\begin{array}{c}\text { Míf. d'HOUR } \\
\text { Midseason }\end{array}$ & $\begin{array}{l}\text { (Calot 1864) } \\
\text { Rose Type }\end{array}$ & Carmine Pink
\end{tabular}

MME. de TREYERAN 8.2 (Dessert 1899) Midseason Rose Type Pinkish White

MME. DUCEL 7.9 (Mechin 1880)

Midseason Bomb Type Silvery Pink

MME. EMILE GALLE 8.5 (Crousse 1881)

Late Midseason Rose Type Shell Pink

$\left.\begin{array}{ccc}1 & 5 & 25 \\ \text { to } & \text { to } & \text { to } \\ 4 & 24 & 100\end{array}\right\} \begin{array}{r}\text { Divi- } \\ \text { sions } \\ \text { each }\end{array}$

MME. FOREL 7.7 (Crousse 1881) Late Midseason Rose Type

MME. GAUDICHAU 8.2 (Millet 1902) Midseason Semi-double Type

MME. GEISSLER 7.9 (Crousse 1880)

Midseason Rose Type Rose Pink

MME. JULES DESSERT 9.4 (Dessert 1909)

Midseason Rose Type Blush White
Glossy Pink

.40 each

1.25 each

.25 each

.30 each

.25 each

$\begin{array}{lll}4.80 & 3.75 \quad \text { each }\end{array}$

.25 each

$\begin{array}{llll}4.00 & 2.00 & 1.75 & \text { each }\end{array}$ 
MME. LEBON 6.8 (Calot 1888)

Late

Rose Type

MME. LEMOINE 7.7 (Calot 1864)

Late

Rose

MME. LEMOINIER 8.0 (Calot 1865)

Midseason

Rose Type
Cherry Pink

Lilac Rose

Lilac Rose

MME. LOISE MERE 7.7 (Calot 1863)

Late Midseason

Rose Type

Flesh White

MME. SAVREAU 7.7 (Savreau 1906)

Early Midseason Crown Type Lilac White

MLLE. DESBUISSON 7.8 (Crousse 1893)

Late Midseason Semi-rose Type Delicate Pink

MLLE LEONIE CALOT 8.1 (Calot 1861)

Late Midseason Rose Type Delicate Pink

MLLE. RENEE DESSERT 6.5 (Mechin 1880)

Midseason Bomb Type Pure Mauve

MLLE. ROUSSEAU 8.1 (Calot 1886)

Midseason Semi-rose Type

Milk White

MLLE. VAILLANT 7.6 (Calot 1867) Early

Bomb Type

Milk White

MARCELLE DESSERT 8.2 (Dessert 1899)

Midseason Crown Type Milk White

MARCHIONESS OF LANSDOWNE 8.6 (Kelway 1899) Midseason

Rose Type

Light Pink

MARECHAL VAILLANT 7.5 (Calot 1867)
Late
Rose Type
Light Red

MARGUERITE GAUDICHAU 8.1 (Millet 1903) Late

Delicate Pink

MARGUERITE GERARD 8.4 (Crousse 1892)

Midseason Semi-rose Type Pale Pink

MARIE 8.1 (Calot 1868)

Very Late Rose Type Lilac White

MARIE CROUSSE 8.9 (Crousse 1892) Midseason Bomb Type

Salmon Pink

MARIE JACQUIN 8.3 (Verdier) Midseason Semi-double Type

MARIE LEMOINE 8.5 (Calot 1869)
Very Late
Rose Type

Rose White

$\begin{array}{llll}2.80 & 2.00 & 1.75 & \text { each }\end{array}$

.25 each

.25 each

$1.00 \quad .75$ each

$.50 \quad .40 \quad$ each

.25 each

.30 each

Midseason Semi-rose Type Bright Crimson

MARTHA BULLOCK 9.1 (Brand 1907)

Midseason

Rose Type

Rose Pink

$8.00 \quad 6.00 \quad$ each

LARGE ORDERS: Please submit your "want list." On certain items, in quantity, it is often possible for us to quote lower than printed prices. 


\begin{tabular}{|c|c|c|c|c|}
\hline$\because \cdots$ & $\begin{array}{c}1 \\
\text { to } \\
4\end{array}$ & $\begin{array}{l}5 \\
\text { to } \\
24\end{array}$ & $\left.\begin{array}{c}25 \\
\text { to } \\
100\end{array}\right]$ & $\begin{array}{l}\text { Divi- } \\
\text { sions }\end{array}$ \\
\hline 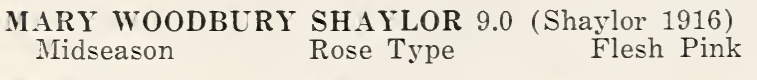 & 8.00 & 7.00 & 6.00 & each \\
\hline $\begin{array}{l}\text { MATHILDE de ROSENECK } 7.9 \text { (Crousse 1883) } \\
\text { Late } \\
\text { Rose Type }\end{array}$ & & & .20 & each \\
\hline $\begin{array}{l}\text { MAUD L. RICHARDSON } 8.5 \text { (Hollis 1904) } \\
\text { Late Lilac Rose Type }\end{array}$ & & 1.75 & & each \\
\hline$\frac{\text { MEISSONIER }}{\text { Midseason }} \begin{array}{c}6.5 \text { (Crousse } 1886) \\
\text { Bomb Type }\end{array}$ Brilliant Crimson & & & .25 & each \\
\hline $\begin{array}{ll}\text { MIGNON } 8.7 \text { (Lemoine } & 1908 \text { ) } \\
\text { Late Midseason } & \text { Rose Type }\end{array}$ & 3.20 & 2.75 & & each \\
\hline 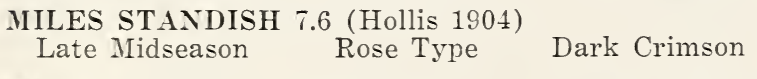 & & & .25 & each \\
\hline $\begin{array}{c}\text { MIREILLE } 7.7 \text { (Crousse 1894) } \\
\text { Very Late } \\
\text { Rose Type }\end{array}$ & & & .20 & each \\
\hline $\begin{array}{l}\text { MISS SALWAY } 7.8 \text { (Kelway 1889) } \\
\text { Early } \\
\text { Crown Tyre }\end{array}$ & 4.00 & 3.50 & 3.00 & each \\
\hline $\begin{array}{l}\text { MODELE de PERFECTION } 7.8 \text { (Crousse } 1875 \text { ) } \\
\text { Late } \\
\text { Rose Type }\end{array}$ & & & .30 & each? \\
\hline $\begin{array}{l}\text { M. BASTIEN-LEPAGE } 7.1 \text { (Crousse 1885) } \\
\text { Late Midseason Crown Type Carmine Rose }\end{array}$ & & & .25 & each \\
\hline $\begin{array}{l}\text { M. BOLCHARLATAINE } 7.1 \text { (Calot 1868) } \\
\text { Late Midseason Semi-rose Type Violet Pink }\end{array}$ & & & .20 & each \\
\hline $\begin{array}{l}\text { M. DUPONT } 8.3 \text { (Calot 1872) } \\
\text { Midseason }\end{array}$ & & & .25 & each \\
\hline $\begin{array}{l}\text { M. KRELAGE } 7.7 \text { (Crousse 1883) } \\
\text { Late Midseason }\end{array}$ & & & .35 & each \\
\hline $\begin{array}{l}\text { M. MARTIN CAHUZAC } 8.8 \text { (Dessert 1899) } \\
\text { Midseason } \\
\text { Semi-rose Type }\end{array}$ & & 1.50 & 1.25 & each \\
\hline $\begin{array}{ll}\text { MONT BLANC } 8.4 & \text { (Lemoine 1889) } \\
\text { Midseason } & \text { Rose Type }\end{array}$ & 2.40 & 2.00 & 1.50 & each \\
\hline $\begin{array}{c}\text { MOSES HULL } 8.2 \underset{\text { Midseason }}{\text { (Brand }} \text { 1907) } \\
\text { Rose Type }\end{array}$ & & 1.00 & & each \\
\hline $\begin{array}{l}\text { MOLNTEBANK (Kelway) } \\
\text { Midseason Anemone Type }\end{array}$ & & 1.00 & .75 & each \\
\hline $\begin{array}{l}\text { MrS. A. G. RUGGLES } 8.3 \text { (Brand 1913) } \\
\text { Late }\end{array}$ & 4.00 & 3.00 & & each \\
\hline $\begin{array}{ll}\text { MRS. CAREW } 7.8 & \text { (Brand 1907) } \\
\text { Midseason } & \text { Rose Type }\end{array}$ & .75 & & & each \\
\hline $\begin{array}{l}\text { MRS. C. S. MINOT } 9.2 \text { (Minot 1914) } \\
\text { Late } \\
\text { Rose Type }\end{array}$ & 28.00 & & & each \\
\hline
\end{tabular}

PLACE ORDERS EARLY: We do not substitute unless instructed by customer to do so. Money promptly returned for any varieties we are sold out of. 
MRS. GEORGE BUNYARD 8.3 (Kelway 1898)

Rose Type

Lilac Rose

NE PLUS ULTRA 5.5 (Miellez 1856)

Early Midseason Rose Type

NEPTUNE 7.6 (Dessert 1905)

Midseason Crown Type

NORFOLK 7.6 (Richardson)

Late Midseason

OPAL 8.5 (Pleas 1908)

Midseason

Rose Type

Violet Rose

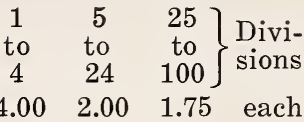

PARADISE 8.7 (Hollis 1907)

Early Midseason Rose Type

Lilac White

Rose Pink

.20 each

PASTEUR 8.4 (Crousse 1896)

Midseason Rose Type

Dainty Pink

$2.00 \quad 1.75 \quad$ each

PERFECTION 8.0 (Richardson 1869)

Very Late

Rose Type

6.00

each

Delicate Pink

Soft Pink

$.50 \quad$ each

$.50 \quad .40$ each

Pale Pink

PETITE RENEE 7.6 (Dessert 1899)

Midseason Semi-double Type

Deep Pink

PHILOMELE 7.7 (Calot 1861)

Midseason Anemone Type Pink and Yellow

$\begin{array}{cccc}\text { PHOEBE CAREY } 8.8 \text { (Brand 1907) } \\ \text { Late } & \text { Rose Type }\end{array}$

PIERRE DUCHARTRE 8.2 (Crousse 1895)

Very Late Rose Type Medium Pink

PIERRE REIGNOUX 7.4 (Dessert 1908)

Early Midseason Semi-rose Type Light Pink

PRESIDENT ROOSEVELT 7.7 (Warnaar 1905)

Midseason Semi-rose Type Deep Red

PRIDE OF ESSEX 8.9 (Thurlow 1916)

Midseason Semi-rose Type

Flesh Pink

PRIMEVERE 8.6 (Lemoine 1907)

Midseason Bomb Type

Yellow

$4.80 \quad$ each

$.90 \quad .75$ each

.25 each

.25 each

$\begin{array}{lll}6.40 & 6.00 \quad \text { each }\end{array}$

$1.75 \quad 1.25$ each

PRINCESS BEATRICE 7.4 (Kelway 1886)

.20 each

Midseason Crown Type Violet Rose

PRINCESS MAY 7.7 (Kelway)
Midseason Anemone Type Pink and Yellow

$.50 \quad$ each

QUEEN EMMA 7.8 (Unknown)

Midseason Rose Type Pale Lilac Rose

$.30 \quad$ each

QUEEN VICTORIA 7.2 (Kelway)

Midseason Crown Type

White

.20 each

LARGE ORDERS: Please submit your "want list." On certain items, in quantity, it is often possible for us to quote lower than printed prices. 
RAOUL DESSERT 9.0 (Dessert 1910) Late Midseason Rose Type

Mauve Pink
Midseason

Rose Type

ROSETTE 8.8 (Dessert 1918)

Early Midseason Semi-rose Type

ROSY DAWN 7.1 (Pleas 1909)

Midseason
Brilliant Pink

Silvery Flesh

.75

Rose Pink

R. P. WHITFIELD 8.0 (Richardson)
Late
Rose Type
Hydrangea Pink

RUBENS 6.8 (Delache 1854)

Early Midseason Semi-double Type Dark Crimson

.25 each

SARAH BERNHARDT 9.0 (Lemoine 1906)

Late Midseason Semi-rose Type Apple Blossom Pink A special offering of one of the World's Finest and most generally satisfactory varieties. Place order early. Orders will be filled in order of receipt until our supply of Sarah Bernhardt is exhausted. This variety sold only in assortment with other varieties.

SIR JOHN FRENCH (Kelway 1915)

SOLANGE 9.7 (Lemoine 1907) Late Rose Type

Deep Pink

$5.00 \quad 4.00 \quad 3.50 \quad$ each

$\begin{array}{llll}4.00 & 2.50 & 2.00 & \text { each }\end{array}$

SOLFATARE 7.6 (Calot 1861)

Midseason Crown Type White, Center Yellow

SOUV. de FRANCOIS RUITTON 7.8 (Riviere 1908) Late Midseason Rose Type Dark Crimson

$.75 \quad .60$ each

SOUV. de L' EXPO. de BORDEAUX 6.4 (Dessert 1896) Midseason

Bomb Type

Violet Red

each

$2.00 \quad 1.75 \quad 1.50 \quad$ each

SOUV. de L' EXPO. UNIVERSELLE 7.6 (Calot 1867) Late Rose Type

Deep Pink

$\begin{array}{ccc}\text { SOUV. de LOUIS BIGOT 9.1 (Dessert } & 1913 \text { ) } \\ \text { Midseason } & \text { Rose Type }\end{array}$ $\begin{array}{lll}4.80 & 4.00 \quad \text { each }\end{array}$

STANLEY 8.0 (Crousse 1879)

Midseason Rose Type Light Pink

SULPHUREA 6.6 (Lemon 1830)

Ey Midseason Crown Type White, Yellow Center

.20 each

.20 each

UMMER DAY 7.8 (Kelway 1895-6)

Late Rose Type Cream White

SUZANNE DESSERT 7.7 (Des. and Mech. 1890)

Midseason Semi-rose Type China Pink

SUZETTE 8.2 (Dessert 1911)

Midseason Semi-rose Type Bengal Rose

$.75 \quad .50 \quad$ each

.20 each

$.75 \quad$ each

$.50 \quad .40$ each

.35 each

PLACE ORDERS EARLY: We do not substitute unless instructed by customer to do so. Money promptly returned for any varieties we are sold out of. 
T. B. TERRY 8.3 (Pleas 1909) Midseason Rose Type

THERESE 9.8 (Dessert 1904) Midseason Rose Type

TORQUEMADA 7.1 (Kelway 1895) Midseason

Rose Type

TOURANGELLE 9.4 (Dessert 1910) Late Midseason

\begin{abstract}
Rose Type
\end{abstract}

\section{BERLIN, MARYLAND}

TRIOMPHE de L' EXPO. de LILLE 7.8 (Calot 1865) Midseason Rose Type Pale Pink

TRIOMPHE du NORD 6.0 (Miellez 1850)

Midseason Bomb Type Carnation Pink $\left.\begin{array}{ccc}1 & 5 & 25 \\ \text { to } & \text { to } & \text { to } \\ 4 & 24 & 100\end{array}\right\} \begin{aligned} & \text { Divi- } \\ & \text { sions }\end{aligned}$

$\begin{array}{ccc}4 & 24 & 100 \\ 3.50 & 3.00 & \text { each }\end{array}$

UMBELLATA ROSEA 7.4 (Dessert 1895)

\begin{tabular}{ccc}
\hline Very Early & Rose Type & Pink and Cream \\
VAN DYCK & 6.5 & (Crousse 1879) \\
Midseason & Crown Type & Clear Pink \\
VICTOIRE & &
\end{tabular}

$\begin{array}{llll}3.20 & 2.25 & 2.00 & \text { each }\end{array}$

Violet Rose

Clear Pink

$.20 \quad$ each

$2.80 \quad 2.25 \quad 2.00 \quad$ each

.25 each

.20 each

.25 each

.20 each

$2.80 \quad 2.25 \quad 2.00 \quad$ each Midseason Dark Crimson

VILLE de NANCY 7.1 (Calot 1872)
Late
Rose Type
Carmine Rose

VIRGINIE 7.6 (Calot 1858)

Midseason Semi-rose Type Light Pink

VIRGO MARIA 7.0 (Calot 1859)
Late Midseason
Bomb Type

Pure White

VISCOUNTESS FOLKESTONE 7.6 (Barr 1899)
Midseason
Rose Type
Clear White

.40

.20 each

VOLCAN 6.5 (Lemoine 1898)

Midseason Bomb Type

Dazzling Red

WALTER FAXON 9.3 (Richardson 1904) Midseason Semi-rose Type Salmon Rose Pink

WASHINGTON 6.4 (Guerin 1850) Midseason Bomb Type

Tyrian Rose

WELCOME GUEST 7.8 (Hollis 1904) Midseason Semi-double Type

WILLIAM PENN 7.9 (Brand 1907) Midseason Semi-rose Type

Bright Rose

.20 each

.20 each

each

$1.00 \quad .75$ each

$\begin{array}{lll}4.00 & 3.00 \quad \text { each }\end{array}$

.20 each

$.60 \quad .50 \quad$ each

each

.20 each

ZOE CALOT 7.0 (Miellez 1855)

Midseason Rose Type Soft Pink

\section{Peonies To Color}

PINK

RED

WHITE AND FLESH SHADES

YELLOW SHADES

ASSORTED

$\begin{array}{ll}.15 & \text { each } \\ .18 & \text { each } \\ .15 & \text { each } \\ .18 & \text { each } \\ .15 & \text { each }\end{array}$

LARGE ORDERS: Please submit your "want list." On certain items, in quantity, it is often possible for us to quote lower than printed prices. 


\section{Japanese Peonies}

AK ALU 8.1

ALMA 8.5 (Shaylor 1916)

ATTRACTION 8.2 (Holihs1906) Midseason

CASHMiERE (Chiyokagami)

FLASHLIGHT 8.2 (Hollis 1906) Early

GERALDINE (Kelway)

Midseason

GOLDEN NUGGET 7.4 (Pleas 1913)

GYPSY 8.0 (Hollis 1904)

INNOCENCE 8.3 (Hollis 1904)

KING OF ENGLAND 8.6 (Kelway 1902)

LABOLAS 7.6 (Kelway)

MARGARET ATWOOD 8.7

SUNBEAM 8.3 (Hollis 1904)

Midseason

THE DRAGON (Wallace)

TORPILLEUR 8.0 (Dessert 1920)
Carmine

$\left.\begin{array}{ccc}1 & 5 & 25 \\ \text { to } & \text { to } & \text { to } \\ 4 & 24 & 100\end{array}\right\} \begin{aligned} & \text { Divi- } \\ & \text { sions }\end{aligned}$

$2.40 \quad 2.00 \quad$ ecah

Pink:

Tyrian Rose

Light Tyrian Rose

Carmine Violet

1.00 each

$\begin{array}{lll}4.00 & 3.00 \quad \text { each }\end{array}$

$1.00 \quad .75$ each

3.20

each

Orange

Tyrian Rose

$2.50 \quad 2.00 \quad$ each

Lilac Rose

1.50 each

$\begin{array}{lll}4.00 & 3.00 \quad 2.50 & \text { each }\end{array}$ Carmine

each

White

2.50

each

$8.00 \quad 7.00 \quad$ each

Rose Pink

1.00 each

each

Brilliant Crimson

each

\section{Single Peonies}

AUSTIN CHAMBERLAIN 7.5 (Kelway 1909) Early

Amaranth Red

BLACK PRINCE 8.5 (Thurlow 1915)

Dark Garnet

CARNOT 7.7 (Dessert 1913)

CLAIRETTE 8.1 (Dessert 1905)

Carmine

each

each

$1.00 \quad$ each

White 
COUNTESS OF WARWICK (Kelway) Early

DOROTHY 7.5 (Kelway 1898)

Midseason

DUCHESS OF SUTHERLAND 7.7 (Kelway 1898)

ETIENNE DESSERT (Dessert)

FLAG OF TRUCE 6.8 (Kelway 1900)

LA FIANCEE 8.4 (Dessert 1902) Very Early

LA FRAICHEUR 8.1 (Dessert 1902)

LETITIA (Kelway)

MADELEINE GAUTHIER 8.5 (Dessert 1908)

MRS. KEY 7.9 (Terry)

Early

Glowing Crimson

PEREGRINA (Unknown)
Very Early

PIOU PIOU (Kelway)

PRIDE OF LANGPORT 8.9 (Kelway 1909)

ROSY DAWN 8.5 (Barr)

Early

SILVER CUP (Kelway 1909)

STANLEY 7.8 (Kelway 1890)

Early Midseason

Dark Crimson

THE MOOR 7.8 (Barr)

Very Early

Purplish Garnet

THE QUEEN 8.3 (Kelway 1902)

WHITLEYI MAJOR 8.5

WILBUR WRIGHT 8.0 (Kelway 1909)
Flesh Pink

Red

Soft Pink

Snow White

$\left.\begin{array}{ccc}1 & 5 & 25 \\ \text { to } & \text { to } & \text { to } \\ 4 & 24 & 100\end{array}\right\} \begin{aligned} & \text { Divi- } \\ & \text { sions }\end{aligned}$

$\begin{array}{lll}2.50 & 2.00 \quad \text { each }\end{array}$

1.00 each

1.00 each

$2.00 \quad 1.75 \quad$ each

$1.00 \quad$ each

$\begin{array}{lll}1.00 \quad .90 & \text { each }\end{array}$

$1.00 \quad$ each

$.60 \quad .50$ each

$2.00 \quad$ each

$1.00 \quad$ each

1.00 each

$.75 \quad .60$ each

$\begin{array}{llll}4.80 & 3.50 & 3.00 & \text { each }\end{array}$

$1.00 \quad$ each

1.00 each

$.50 \quad .40 \quad$ each

$.60 \quad .50$ each

$.60 \quad .50$ each

Blush White

Blush White

1.00 each

$.90 \quad .75$ each

Deep Maroon

LARGE ORDERS: Please submit your "want list." On certain items, in quantity, it is often possible for us to quote lower than printed prices. 


\section{Plant Peonies for Cut-Flower Profits}

About 3,600 to 5,000 plants required to set an acre. We often sell from $\$ 1,000$ to $\$ 1,500$ worth of cut-flowers in one season from an acre of Peonies. You should do as well. Plant an acre.

\section{Planting ,Suggestions}

Peonies should be planted in the fall, at any time from September first until the ground freezes too dcep to allow planting. and they succeed best in good quality, well drained garden soil, such as would produce a good crop of potatoes.

Place roots in their natural position, two to four feet apart (for permanent cut-flower planting, rows may be four feet apart, plants three feet apart in the row, or $3 \frac{1}{2} \times 3_{1 / 2}^{1 / 2}$ feet will be preferred by some) with new eyes two inches below the surface of the ground. Many growers plant as close as 3 X 3 feet.

An application of raw ground bone (bone meal) thoroughly mixed with the soil will supply sufficient plant food for a rear or two. A top dressing of hardwood ashes would assist in quick root derelopment.

Soil should be well prepared by plowing or spading and cultivating to a depth of eight inches or more and allowed to settle for a few days prior to setting the roots.

On some types of soil (usually not necessary), a light covering of coarse well rotted stable manure or leaves or other mulch for the first winter will prevent the newly set roots from heaving out because of alternate freezing and thawing.

Remove covering from directly over the plants, in early spring, before Peonies come through the ground. When Peonies are up a few inches, cultivate thoroughly and often throughout the growing season.

\section{FOR THE TRADE NEWS}

We recommend The American Florist, America's Oldest Floricultural Trade Paper. Published every Thursday. \$1.00 per year, \$2.00 for three years.

Mail your subscription to 60 West Washington Street, Chicago, Illinois.

\section{FOR ALL WHO GROW FLOWERS}

We recommend The Flower Grower. Published monthly. "The Magazine With a Mission." Edited, published and owned by Madison Cooper.

A publication that is all that its name implies. But it is also much morc.

Those who are interested in outdoor activities and who aspire to better living conditions; more beautiful surroundings; and a better outlook on life; will find many useful hints, suggestions. facts and information.

Subscription price: $\$ 2.00$ per year; 3 years, $\$ 4.50 ; 5$ years, $\$ 6.00$; one copy 20c.

Mail your subscription direct to: The Flower Grower, Calcium, N. Y. 
MEMORANDUM 


\section{Decoration Day Varieties}

These varieties, in many localities, bloom sufficiently early for Decoration Day sales and are therefore considered very valuable for commercial cut-flower plantings where early blooming is important.

ALEXANDRE DUMAS 7.1 (Guerin 1862)

Light Violet-Rose Crown Type. One of the older varieties, still planted in quantity for cutflowers. Blooms are of medium size, with guards and center of bright violet-rose and narrow chamois-colored petals mingled with the center and forming the collar. The plant is of spreading habit and is an exceptionally free and reliable bloomer.

EARLY.

DUCHESSE DE NEMOURS 8.1 (Calot 1856)

Yellow-White

Crown 'Iype. Opens with center tinted a beautiful sulphur- yellow which later faces to white. Pleasant odor. Vigorous, upright grower and one of the very best bloomers. Blooming as it does, about two days later than Festiva Maxima, makes it a very valuable kind to use in combination with Festiva Maxima. This is one of the very best commercial cut-flower Peonies and no grower can aftord to leave it out of his planting. We recommend a good portion of any commercial planting to be of Duchesse de Nemours.

EARLY.

EDULIS SLPERBA 7.6 (Lemoine 1824)

Deep Pink Crown Type. Strong, hardy grower; prolific bloomer; very fragrant. Bright rose pink and generally known in the cut-flower market as "Decoration Day rink." A good kee' sr and shi's well. Over cne hundred years ola but still leading in the field of reliable early deep pinks.

EARLY.

FESTIVA MAXIMA 9.3 (Millez 1851)

White

Rose Trpe. Pure white with prominent crimson markings; fragrant. Very sturdy, tall, strong stems; blooming habit very dependable and succeeds wherever Peonies will grow. Thought by many to be the finest white in existence and surely the most popular of all cut-flower Peonies. Hundreds of acres of this variety have been planted for producing commercial cutfiowers for the home and city markets. For serenty six years Festiva Maxima has maintained its leadership.

EARLY;

FLORAL TREASURE 7.5 (Rosefield 1900)

Salmon Pink

Rose Type. Delicate salmon-pink. Center petals are loose and spread just enough to show the carpels of light greenish-yellow, tipped white, giving a fresh, dainty color effect. Flowers are fragrant and on well established plants are very large and perfectly formed. Strong, tall grower. Desirable for landscape and commercial cut-flowers.

EARLY MIDSEASON.

MME. DE VERNEVILLE 7.9 (Crousse 1885)

White

Bomb Type. Pure white with petals carnine tipped. It has the true June Rose fragrance and is one of the most charming of all varieties. This is an old established and fully tested Peony and for dependability for cut-flowers and general planting, is unusually valuable.

EARLY.

UMBELLATA ROSEA 7.4 (Guerin 1895)

Light Pink Rose Type. Shell pink with center petals a creamy white. Strong upright grower and profuse bloomer. Comes in bloom earlier than any other Peony of the "so called" Chinensis class which makes it extremely valuable for cutting in time for Decoration Day market. A profitable variety. VERY EARLY.

\section{MOST POPULAR CUT-FLOWER RED}

FELIX CROUSSE 8.4 (Crousse 1881) Red Bomb Type. Vigorous grower; spreading habit; blooms freely. Bright red; pleasant odor. The most popular and satisfactory of all red Peonies for cut-flower purposes, which can be purchased at a low price. Good for general planting and landscape work. We recommend Felix Crousse without reservation.

MID-SEASON.

NOTE: Most of the above varieties, can be furnished in lots of $1,000,5,000$, and 10,000. For prices, see "General List," in this price list. 
DAYLILIES

GLADIOLI

DELPHINIUMS

\section{FRANDAMA GARDENS \\ PEONIES AND IRISES \\ HOLLAND BULBS}

425 Ashbourne Road (rear), Elkins Park, Pa. May 31, 1927.

Mr. A. H. Hardesty,

Harmel Peony Company,

Berlin, Md.

Dear Mr. Hardesty:-

The return trip has been made safely and without incident. It was five very pleasant days that I have passed with you. I can not refrain from going on to add that I was delighted with everything that I found in Berlin and at your peony farm. You have a fine big stock in excellent condition, and it is being cared for well, too. In fact, in all my experience I have not found a big lot of peonies that has been managed better. And my experience with this flower dates back quite a way, fifteen years indeed. I have been associated, as you know, with some of the most eminent and successful growers in the country. I want to commend the painstaking accuracy you exercise in having everything just right. And it is delightful to know that you have progress ahead of you, for along with the splendid stock of the good standard varieties you have the beginnings of many of the most prominent new ones the increase of which your patrons can look forward to. I am very grateful for the kind entertainment given me.

Yours cordially,

Frank B. Meyer. 